\title{
Langmuir Films of Anthracene Derivatives on Liquid Mercury II: Asymmetric Molecules
}

\section{Tamam}

Department of Physics, Bar-Ilan University, Ramat-Gan 52900, Israel

\section{H. Kraack}

Department of Physics, Bar-Ilan University, Ramat-Gan 52900, Israel

\section{E. Sloutskin}

Department of Physics, Bar-Ilan University, Ramat-Gan 52900, Israel

\section{B. M. Ocko}

Department of Physics, Brookhaven National Laboratory, Upton, New York 11973

\section{P. S. Pershan}

Department of Physics and DEAS, Harvard University, Cambridge, Massachusetts 02138

\section{E. Ofer}

Department of Physics, Bar-Ilan University, Ramat-Gan 52900, Israel

\author{
M. Deutsch* \\ Department of Physics, Bar-Ilan University, Ramat-Gan 52900, Israel
}

Received: June 23, 2006; In Final Form: November 28, 2006

\begin{abstract}
The structure and phase sequence of liquid-mercury-supported Langmuir films (LFs) of anthrone and anthralin were studied by surface tensiometry and surface-specific synchrotron X-ray diffraction. In the low-coverage phase the molecules are both side-lying, rather than the flat-lying orientation found for anthracene and anthraquinone. In the high-coverage phase, the molecules are either standing up (anthrone) or remain sidelying (anthralin) on the mercury surface. In contrast with the symmetric anthracene and anthraquinone, both high-coverage phases exhibit long-range in-plane order. The order is different for the two compounds. The structural details, the role of molecular symmetry, the oxygen side groups, and the asymmetry-induced dipole moments are discussed.
\end{abstract}

\section{Introduction}

The previous paper ${ }^{1}$ detailed the reasons for undertaking the present study, discussed the measurement methods, and presented the results obtained for mercury-supported Langmuir films (LFs) of anthracene and anthraquinone molecules, the structure of which is symmetric relative to both the long and short axes of the molecules.

We present here surface tensiometry and X-ray diffraction results for two asymmetric derivatives of anthracene: anthrone and anthralin, shown in Figure 1. These molecules lack mirror symmetry with respect to the long axis of the molecules, although the mirror symmetry with respect to the central short axis is preserved. The planar anthrone, $\mathrm{C}_{14} \mathrm{H}_{10} \mathrm{O}$, is a keto form of 9-anthranol. Anthralin, $\mathrm{C}_{14} \mathrm{H}_{10} \mathrm{O}_{3}$ has two additional oxygens. Both isomers of the keto-enol equilibria shown in Figure 1 can be isolated, but the keto form is the stable one because of the hydrogen $\mathrm{OH} \cdots \mathrm{O} \cdots \mathrm{OH}$ bonds between the $\mathrm{C}=\mathrm{O}(\mathrm{H})$ moieties. ${ }^{2}$ In addition to presenting the results obtained for the

* Corresponding author. E-mail: deutsch@mail.biu.ac.il. (a)
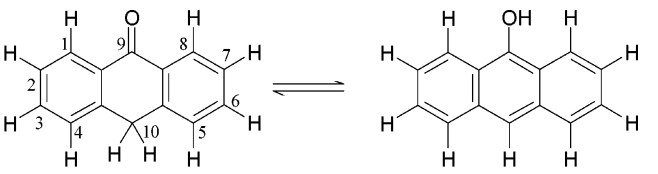

(b)
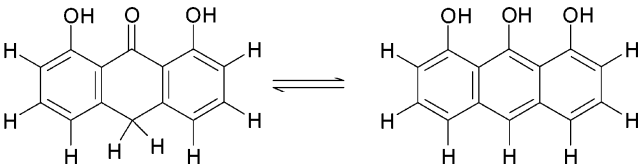

Figure 1. Molecular structure of the molecules studied. (a) Anthrone, showing the numbering convention of the carbons; (b) anthralin. Arrows indicate keto-enol equilibria. The left side is the keto, which has two hydrogen atoms at the 10 position. The right side is the enol form, where only one hydrogen atom is bound at the 10 position, and the second hydrogen is bound to the oxygen atom at the 9 position of the molecule.

asymmetric anthrone and anthralin molecules, we also discuss these results together with those obtained for the symmetric antharcene and anthraquinone molecules in the previous paper. ${ }^{1}$ 


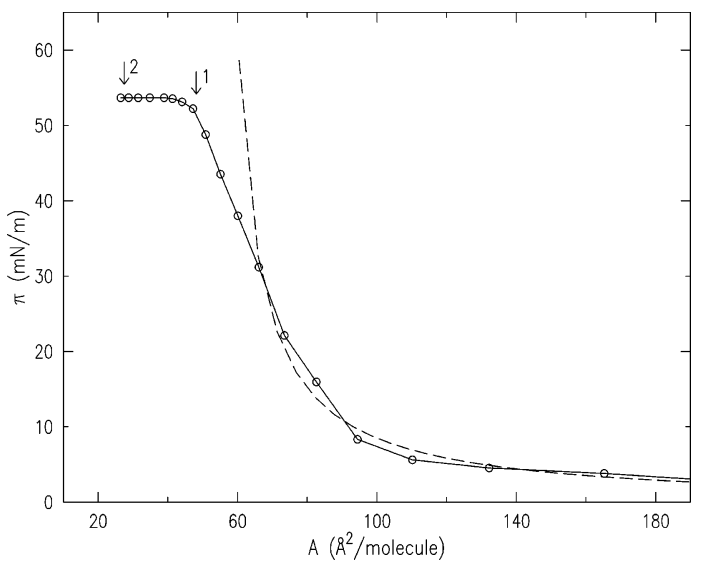

Figure 2. $\pi-A$ isotherm of a LF of anthrone on mercury (circles and solid line). The dashed line is a fit by the Vollmer equation, yielding an exclusion area of $A_{0}=53 \AA^{2} /$ molecule. The arrows mark the coverages where $\mathrm{X}$-ray measurements were carried out.

\section{Results}

A. Anthrone: Isotherm. The measured pressure-area, $\pi-A$, isotherm of a LF of anthrone on mercury at room temperature, $T=24{ }^{\circ} \mathrm{C}$, is shown in Figure 2. A decrease of $A$ from $\sim 300$ $\AA^{2} /$ molecule to $\sim 95 \AA^{2} /$ molecule results in only a few millinewtons per meter increase in $\pi$, as expected for a dilute $2 \mathrm{D}$ gas of molecules. For $A<95 \AA^{2} /$ molecule, however, a steep rise occurs in $\pi$, from $\sim 8.5 \mathrm{mN} / \mathrm{m}$ at $A \approx 95 \AA^{2} /$ molecule to $\sim 51$ $\mathrm{mN} / \mathrm{m}$ at $A \approx 48 \AA^{2} /$ molecule. In contrast with other LFs on mercury, the fast-increasing region at $95 \geq A \geq 48 \AA^{2} /$ molecule of the isotherm can be only poorly fitted by the Vollmer equation $^{1}$ (dashed line in Figure 2, fitted to points in the range $59 \leq A \leq 600 \AA^{2} /$ molecule), and yields an exclusion area of $A_{0}=(53 \pm 1.5) \AA^{2} /$ molecule. The area of a flat-lying anthrone molecule should be equal to, or larger than, that of the anthracene, $A_{\text {calcd }}^{\mathrm{lw}}=l \times w \approx 12 \times 7.5=90 \AA^{2} /$ molecule, obtained in the previous paper. ${ }^{1}$ Here, $l$ is the length and $w$ is the width of the molecule. The fact that the exclusion area obtained here is much smaller indicates that at least near the steep rise the molecules are not lying flat on the surface. In fact, $A_{0}$ is closer to the cross section of the anthracene standing on its side: $A_{\text {calcd }}^{\text {ld }}=l \times d \approx 12 \times 3.8=45 \AA^{2} /$ molecule, where $d$ is the thickness of the anthracene molecule normal to its plane. However, the unit cell dimensions obtained from the grazing-incidence diffraction measurements of anthralin in the side-lying phase, discussed below, indicate that the presence of the oxygen moieties increases the effective thickness of the anthralin molecules by $\sim 0.7 \AA$ over that of anthracene. Assuming that this conclusion also holds for anthrone, which also has an oxygen moiety, this results in a calculated $A_{\text {calcd }}^{\text {ld }}=$ $l \times d \approx 12 \times(3.8+0.7)=54 \AA^{2} /$ molecule for a side-lying anthrone molecule, in agreement with the exclusion area of $A_{0}$ $=53 \AA^{2} /$ molecule obtained here from the Vollmer equation.

As $A$ is reduced below $48 \AA^{2} /$ molecule, a plateau is observed in the isotherm. Previous studies show that such plateaus represent coexistence regions between different-structure phases. ${ }^{3-7}$ Continuing the line of thought presented above, this plateau may represent a coexistence between phases of sidelying and standing-up molecules. The latter phase is also found in alkyl chain molecules. ${ }^{3,5-7}$ These suggestions, derived here from the isotherm alone, are supported by the X-ray measurements discussed in the next two subsections.

B. Anthrone: Surface-Normal Structure. Figure 3a shows a set of Fresnel-normalized X-ray reflectivity (XR) curves measured (open circles) at room temperature, $T=24{ }^{\circ} \mathrm{C}$, at the
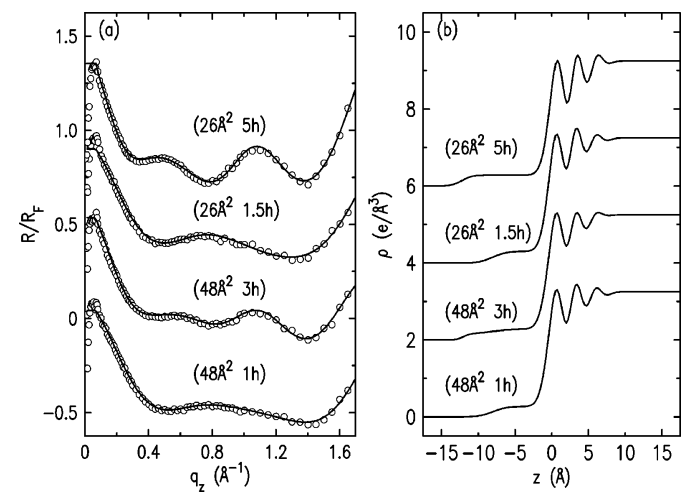

Figure 3. (a) Measured Fresnel-normalized X-ray reflectivity curves (open circles) for anthrone at the nominal area (per molecule) and time (since deposition) indicated. The box model fits (lines) are also shown. (b) The surface-normal electron density profiles obtained from the fits, corresponding to the lines in part a. Curves in parts $a$ and in $b$ are shifted from each other by 0.4 and 2 , respectively, for clarity.

listed nominal molecular area $A$ and at the listed time after deposition of the LF. Fits to the box model discussed in the previous paper $^{1}$ (lines) yield the electron density profiles plotted in the corresponding lines in Figure 3b, where the mercuryanthrone interface is taken as the $z$-axis origin, $z=0 \AA$.

Curves identical to the long-time ones shown in Figure 3 were also observed at $T=4.7^{\circ} \mathrm{C}$ and $T=7.5^{\circ} \mathrm{C}$ for the same coverages, provided that $\sim 2.5 \mathrm{~h}$ or more have passed since the LF was spread. The XR curves measured for four different samples, each studied at 2-3 coverages and 2-3 temperatures, lead to the conclusion that at high coverages the XR curves vary only with the time elapsed since the spreading of the film, but not with temperature. At low coverages, the dominant variation is with time, although a weak dependence on temperature cannot be ruled out.

We now discuss in details the XR results for low $A$, marked with arrow no. 1 in the isotherm in Figure 2, and high $A$, marked with arrow no. 2 .

1. Low Coverage. The modulation period in the XR curves at the nominal coverage of $A=48 \AA^{2} /$ molecule, slightly below the onset of the plateau in the isotherm, is found to decrease with increasing time following the spreading of the film. This corresponds to an increase in the thickness of the layer with time. The fit of the XR curve measured $1 \mathrm{~h}$ after film spreading yields a uniform LF of a (fixed) $8 \AA$ thickness and a (fitted) electron density of $\rho=(0.28 \pm 0.02) \mathrm{e}^{\circ} \AA^{3}$. Attempts to fit the XR curve with a thickness of $d \approx 4 \AA$ failed. This thickness is close to the width of the molecule and therefore supports the suggestion above that the molecules at this coverage are sidelying: the molecular plane is roughly normal to, and the molecular long axis is roughly parallel to, the mercury surface. Three hours after deposition the period of the modulations is shorter, $\Delta q_{z} \approx 0.48 \AA^{-1}$, yielding an estimate of $d=2 \pi / \Delta q_{z} \approx$ $13 \AA$ for the layer thickness. The fact that this value is close to the $\sim 12 \AA$ length of a molecule implies that at that time a considerable fraction, if not all, of the molecules stand up. To allow for the coexistence between a side-lying (SL) phase and a standing-up (SU) phase (which is observed to also be the single, uniform, phase in the high-coverage regime, discussed below), we adopt a model used in previous studies for similar coexistence regions. ${ }^{3,5-7}$ The LF is represented by two layers. The lower layer is $8 \AA$ thick and comprises the side-lying molecules plus the lower part of the standing-up molecules. The upper layer, of thickness $\sim 4 \AA$, comprises the upper part of the standing-up molecules. While the lower layer is close-packed, 
TABLE 1: Fit Parameters of the Anthrone XR Curves, Measured as a Function of the Time Elapsed since the Spreading of the Film, at Two Nominal Coverages $A^{a}$

\begin{tabular}{|c|c|c|c|c|c|c|}
\hline \multirow[b]{2}{*}{$\begin{array}{c}\text { time } \\
\text { (hours) }\end{array}$} & \multicolumn{2}{|c|}{ SL } & \multicolumn{2}{|c|}{ SU } & \multirow[b]{2}{*}{$\begin{array}{l}\sigma_{\mathrm{LF}} \\
(\AA)\end{array}$} & \multirow[b]{2}{*}{$\begin{array}{l}\sigma_{\mathrm{Hg}} \\
(\AA)\end{array}$} \\
\hline & $\begin{array}{c}d_{1} \\
(\AA)\end{array}$ & $\begin{array}{l}\text { cov. } \\
(\%)\end{array}$ & $\begin{array}{c}d_{2} \\
(\AA)\end{array}$ & $\begin{array}{l}\text { cov. } \\
(\%)\end{array}$ & & \\
\hline \multicolumn{7}{|c|}{ Nominal area $A=48 \AA^{2} /$ molecule } \\
\hline 1 & 8 & 100 & & & 1.5 & 1.2 \\
\hline 2 & 8 & 56 & 12.2 & 44 & 0.7 & 1.2 \\
\hline 3 & 8 & 42 & 12.2 & 58 & 0.8 & 1.2 \\
\hline $6.5^{b}$ & 8 & 30 & 12.1 & 70 & 1.0 & 1.0 \\
\hline 10 & 8 & 30 & 12.1 & 70 & 1.0 & 1.2 \\
\hline \multicolumn{7}{|c|}{ Nominal area $A=26 \AA^{2} /$ molecule } \\
\hline 1.5 & 8 & 100 & & & 1.5 & 1.1 \\
\hline 5 & & & 12.5 & 100 & 1.1 & 1.1 \\
\hline 9.5 & & & 12.3 & 100 & 1.1 & 1.1 \\
\hline
\end{tabular}

${ }^{a}$ The percent coverage ("cov.") and layer thickness $\left(d_{1,2}\right)$ are listed for the standing-up (SU) and side-lying (SL) phases. The roughness of the $\mathrm{LF}$-air interface $\left(\sigma_{\mathrm{LF}}\right)$ and $\mathrm{LF}-$ mercury interface $\left(\sigma_{\mathrm{Hg}}\right)$ are also listed. Uncertainties in $d_{2}$ are \pm 0.1 , in $\sigma_{\mathrm{LF}} \pm 0.3 \AA$, and in $\sigma_{\mathrm{Hg}} \pm 0.2 \AA$. ${ }^{b}$ Measured at $T=4.5^{\circ} \mathrm{C}$.

the upper layer is not, unless all molecules stand up. The ratio of the fitted electron densities of the upper and lower layers is, therefore, the fraction of the LF's total area covered by standingup molecules. This method effectively averages coherently over the coexisting different-phase domains. Such averaging is supported by the good contrast ${ }^{8}$ in the $R / R_{F}$ fringes, observed in the $3 \mathrm{~h}$ old XR curve in Figure $3 \mathrm{a}$, and by all previous fits of coexisting phases in several other LFs on mercury. ${ }^{3,5-7}$ The fit to this model in Figure 3a yields a thickness of (12.2 \pm 0.1$)$ $\AA$ for the standing-up part of the LF, close to the $\sim 12 \AA$ length of the molecule, and an electron density of $\rho=(0.16 \pm 0.01)$ $\mathrm{e} / \AA^{3}$, which indicates a fractional coverage of $(58 \pm 8) \%$ by the standing-up phase. As shown in Table 1, fits to XR curves measured $3.5 \mathrm{~h}$ (low $T$ ) and $7 \mathrm{~h}$ later show that the fractional coverage by the SU phase increased to $(70 \pm 6) \%$, and remained stable with time at that value.

For LFs of all molecules studied on mercury to date, including the anthracene and anthraquinone discussed in the previous paper, ${ }^{1}$ the low-coverage phase consists of molecules that lie flat on the high-energy ( $\sigma=486 \mathrm{mN} / \mathrm{m}$ ) surface of mercury. This arrangement reduces the surface's free energy by using the smallest possible number of molecules to cover the surface. It is therefore surprising that the anthrone is found to lie on its side. This alignment does not provide the largest possible contact area with the subphase, and, consequently, does not yield the largest possible reduction in free energy. Because such an alignment does not occur for anthracene, it is very plausible that the anthrone's side alignment is due to the oxygen, the contact of which with the surface is thus maximized, presumably overcompensating for the incomplete free energy reduction due to the side-lying alignment. The better contact between the parallel-aligned rings of adjacent side-lying molecules, as compared to flat-lying molecules, may also contribute to the reduction in the system's free energy. However, the major contribution has to come from the $\mathrm{Hg}-\mathrm{O}$ contact.

A possible reason for the preference of the molecules to stand up is that in the SL phase the charged oxygens of adjacent molecules are close to, and thus repulse, each other. The presence of the oxygen on one side of the molecule only induces a strong electric dipole moment of $3.3 \mathrm{D}^{9}$ in the direction of the short molecular axis of the molecule, running through carbons 9 and 10 in Figure 1. Because adjacent molecules in the SL phase have their oxygens on the same side of the molecule (the one touching the mercury surface), repulsion between the parallel-aligned dipoles ensues. In contrast, when the molecules stand up it is possible to have the oxygens of adjacent molecules point in opposite directions, aligning the dipoles antiparallel to each other. If the nearest-neighbor positions are anisotropic, then this can yield a net dipolar attraction that would reduce the free energy. Several in-plane structural motifs are possible with such pairs of standing-up antiparallel molecules, for example, stripe phases, pair-decorated lattice points, and so forth. In principle, the packing can be refined from grazing-incidence diffraction (GID) patterns, when these show diffraction peaks corresponding to long-range inplane order. However, the lack of such GID peaks for the lowcoverage layer, discussed below, precludes a definite determination of the molecular packing, and relative dipole orientation, in the present phase.

2. High Coverage. The X-ray measurements at the highcoverage phase, presented in Figure 3, were carried out at a nominal $A=26 \AA^{2} /$ molecule, near the lowest- $A$ end of the plateau, where $A \approx A_{0} / 2$. Figure 3 shows two XR curves, measured on the same sample 1.5 and $5 \mathrm{~h}$ after deposition. Fits to XR curves taken $5 \mathrm{~h}$ (shown) and $9 \mathrm{~h}$ (not shown) after deposition show a stable film of thickness $d=(12.4 \pm 0.1) \AA$ and an electron density of $\rho=(0.28 \pm 0.01) \mathrm{e} / \AA^{3}$ (Table 1$)$. As discussed above, these values correspond well to a uniform monolayer of molecules oriented with their long axis normal to the mercury surface. At short times (1.5 h) a side-lying phase is observed, as indicated by the fit to the corresponding XR curve in Figure 3, and the resultant values listed in Table 1. Because a $100 \%$ coverage by the SU phase is achieved with time, clearly this is the stable phase, and the SL phase is an unstable transient.

C. Anthrone: Surface-Parallel Structure. The GID scans for anthrone spanned the range $0.30 \leq q_{\|} \leq 1.73 \AA^{-1}$ and were done in several separate scans, rather than a single scan, with the trough moved between these scans to expose a fresh surface area, thus minimizing beam damage effects. For all nominal coverages studied, except for $A \leq 32 \AA^{2} /$ molecule, no GID peaks were observed either at room temperature, $T=24{ }^{\circ} \mathrm{C}$, or at lower temperatures, $T=4.5,7.5,9.5{ }^{\circ} \mathrm{C}$. This indicates that these LFs have only short-range in-plane order. For the nominal coverages of 26 and $32 \AA^{2} /$ molecule, GID peaks are observed only at low temperatures, but not at room temperature. The most extensive set of GID measurements was carried out at a nominal coverage of $A=32 \AA^{2} /$ molecule and $T=7.5{ }^{\circ} \mathrm{C}$. The GID patterns suggest a coexistence of two ordered phases, one with a noncentered rectangular unit cell and the other with a hexagonal packing. We now discuss these results in detail.

Figure 4 shows the GID pattern for anthrone at a nominal coverage of $A=26 \AA^{2} /$ molecule and $T=7.5^{\circ} \mathrm{C}$, measured 4.5 $\mathrm{h}$ after film spreading and $3 \mathrm{~h}$ after cooldown to $T=7.5^{\circ} \mathrm{C}$. Earlier partial scans of the region of the two lowest-order peaks reveal fully developed peaks at $q_{\|}=0.742$ and $0.776 \AA^{-1} \sim 30$ min after cooldown. Repeated partial scans at longer times, and a full scan $6 \mathrm{~h}$ after cooldown, prove that the GID pattern shown in Figure 4 is stable with time.

The three lowest $q_{\|}$peaks can be indexed in a rectangular unit cell of dimensions of $\mathbf{a} \times \mathbf{b}=(8.09 \pm 0.01) \times(8.45 \pm$ 0.01) $\AA^{2}$, with two molecules per unit cell. Thus the X-rayderived area per molecule is $A_{x}=8.09 \times 8.45 / 2=(34.2 \pm$ $0.1) \AA^{2}$. The reflectivity indicates that the molecules are standing up. Note that the area occupied by a vertically aligned anthracene moiety of the anthrone, calculated from its dimensions, should be $\sim 7.5 \times(3.8)=28.5 \AA^{2}$, which is smaller than the $A_{x}=$ $34.2 \AA^{2}$ of the unit cell. As we show below, the slightly larger 

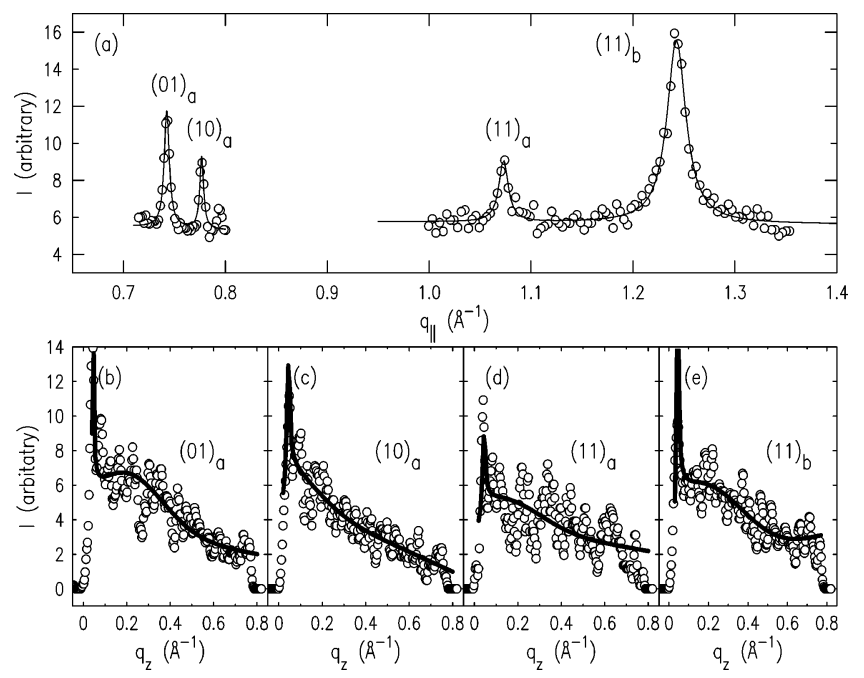

Figure 4. Measured (open circles) (a) GID patterns and $(b-e) B R$ curves, for the SU phase of anthrone at $A=32 \AA^{2} /$ molecule and $T=$ $7.5^{\circ} \mathrm{C}$. The model fits are shown in lines. The BRs correspond to the GID peaks at (b) $q_{\|}=(0.742 \pm 0.002) \AA^{-1}$, (c) $q_{\|}=(0.776 \pm 0.002)$ $\AA^{-1}$, (d) $q_{\|}=(1.073 \pm 0.002) \AA^{-1}$, and (e) $q_{\|}=(1.242 \pm 0.003) \AA^{-1}$. $(. .)_{a}$ and $(. .)_{b}$ denote the rectangular and hexagonal phases, respectively.

$A_{x}$ here is due to a molecular tilt, found in the BR measurements, with a possible contribution from the oxygen moieties. Alternatively, a rotator phase, with molecules in the vertical orientation, would require a molecular area that is somewhat larger than the cross section of a rotating molecule. With a width of $7.5 \AA$ for the anthracene moiety, the cross section of a rotating vertically aligned molecule is at least $\sim \pi(7.5 / 2)^{2}=44.2 \AA^{2} /$ molecule, much larger than the $34.2 \AA^{2} /$ molecule found. Thus, a herringbone-like, or parallel-molecular-plane, ordering ${ }^{10}$ most likely occurs. It is tempting to assign the rectangular unit cell, and the possible herringbone packing, to the existence of a glide (or a mirror) symmetry along one of the axes. Such symmetry was found to dominate the structure of many of the LFs studied to date on aqueous surfaces. ${ }^{10,11}$ This, however, would necessitate the absence of either the (01) or the (10) peaks, depending on whether the symmetry plane is parallel to the $\mathbf{a}$ or $\mathbf{b}$ axis of the unit cell. In our case both $(01)_{a}$ and $(10)_{a}$ are observed, rendering the assumption of a glide symmetry along one of the axes untenable.

The observation of both lowest-order odd $(h+k)$ peaks, $(01)_{a}$ and $(10)_{a}$, leads, therefore, to the conclusion that the unit cell is noncentered. For mercury-supported alkyl thiols, ${ }^{7}$ the headgroups in the SU phase were found to form a similarly noncentered unit cell even though the alkyl moieties of the molecules formed a centered cell. The noncentricity of the headgroup's unit cell was assigned there to the inclusion of a single mercury atom per unit cell into the headgroup's lattice, due to the strong $\mathrm{S}-\mathrm{Hg}$ bond. In contrast, no headgroup exists for the present molecule, and a single unit cell describes the packing of the molecules. Unfortunately, beam damage prevented measuring adequate-statistics GID data at the same position of the sample for all three peaks. Because the monolayer is a coarse $2 \mathrm{D}$ powder, moving the sample to a different position changes the diffracted intensity. Thus, a full refinement, which would have allowed a determination of the position of the molecules within the unit cell and the cause of its noncentricity, as well as ascertain the herringbone packing, could not be carried out.

The fourth peak, at $q_{\|}=1.242 \AA^{-1}$, could not be indexed in the noncentered unit cell above, nor, indeed, were we able to find a unit cell in which all four GID peaks could be indexed.
Thus, a second, coexisting, phase had to be assumed. The single peak of this phase suggests a hexagonal packing, which, in a rectangular representation, has a two-molecule unit cell of dimensions $\mathbf{a} \times \mathbf{b}=(5.84 \pm 0.01) \times(5.84 \pm 0.01) \times \sqrt{3} \AA^{2}$, with an area per molecule of $A_{x}=(29.5 \pm 0.1) \AA^{2} /$ molecule. We have also considered the possibility that this single peak might arise from phases other than hexagonal. One possibility is that the phase is highly tilted, giving rise to peaks far from the $q_{z}=0 \AA^{-1}$ plane, above the $q_{z}$ range scanned by the PSD. However, the XR reveals a monolayer thickness close to that of fully extended, surface-normal molecules. This renders the tilt hypothesis untenable because a high molecular tilt would result in a thinner layer. Moreover, the BR of the $(11)_{b}$, shown in Figure 4e, corresponds to a surface-normal molecule. A single GID peak may also result from 2D smectic-like ordering. However, such ordering seems unlikely for a system that does not exhibit bulk mesophases. Thus, the most appealing physical model is the hexagonal phases.

A rotator phase, very common in the case of hexagonal packing, is highly unlikely in view of the small $A_{x}$ of the present hexagonal phase. However, the fact that the $A_{x}$ of the rectangular phase is larger than that of the hexagonal phase indicates a possible molecular tilt in one or both phases. This is indeed consistent with the BR results presented in the next section.

Although a hexagonal or trigonal in-plane ordering of molecules in organic monolayers is often associated with rotator phases, ${ }^{10,11}$ where the volume occupied by a molecule is cigarlike, phases of molecules that do not incorporate alkyl chains were also found to exhibit such order. One example is cholesterol, the molecular shape of which is plate-like, similar to that of the anthralin. At low coverage cholesterol exhibits a monolayer phase, wherein the long molecular axis is aligned along the surface normal, as it does for the hexagonal phase of the anthrone, and the in-plane order exhibits a trigonal p3 symmetry. At higher coverages, this phase coexists with an order bilayer phase having a centered rectangular unit cell. ${ }^{12}$

The two coexisting hexagonal and rectangular phases differ in both the intensity of their corresponding peaks and in the widths of the peaks. The integrated intensity ratio of the (11) peaks of the two phases is 1:10 in favor of the proposed hexagonal phase. Although this ratio is partly due to the higher degeneracy of the suggested hexagonal phase's peak, and perhaps an additional factor from the slightly different DebyeWaller and structure factors, this ratio indicates that a larger fraction of the area is covered by the hexagonal phase than that covered by the rectangular one.

The widths of the rectangular phase's $(01)_{a},(10)_{a}$, and $(11)_{a}$ peaks range from the diffractometer's resolution limit, $\Delta q_{\|}=$ $0.006 \AA^{-1}$, to $\Delta q_{||}=0.008 \AA^{-1}$. The $(11)_{b}$ peak of the proposed hexagonal phase is considerably broader: $\Delta q_{\|}=0.03 \AA^{-1}$. Thus, the crystallinity of the rectangular phase is considerably higher than that of the proposed hexagonal phase, with the former having a crystalline coherence length of $\xi \geq 1000 \AA$, and the latter $\xi \approx 200 \AA$ only.

Although the GID results clearly indicate here the presence of two coexisting standing-up phases of different in-plane structure, the reasons for this are not clear. Such coexistence usually results from a structural packing frustration of some sort, which is difficult to identify in the present case. We speculate that the coexistence may be related to the orientation of the oxygen moiety of the molecule. The anthrone molecules in the bulk can take, at random, two possible orientations in the molecular plane, transformable to each other by a $180^{\circ}$ rotation around the long molecular axis. ${ }^{14}$ The two phases found 
in the monolayer may reflect phases with randomly and uniformly oriented molecules, with the higher-symmetry hexagonal phase comprising, presumably, the randomly oriented molecules. Packing strains due to the random orientation may also be the cause of the shorter coherence length of this phase, as compared to the orientationally ordered rectangular phase. If indeed the rectangular phase comprises molecules the oxygen groups of which are uniformly oriented, then the larger molecular area of this phase may reflect the need to increase the intermolecular distances to reduce the additional repulsion due to parallel-oriented dipoles induced by the oxygens in the molecule. Further measurements are required to test the validity of these suggestions.

D. Anthrone: Bragg Rods and Molecular Tilt. The measured (open circle) and model fitted (lines) BR's corresponding to the four GID peaks are shown in Figure $4 \mathrm{~b}-\mathrm{e}$. The fits employ the model discussed in detail in ref 15 . The short measuring times dictated by beam damage considerations result in low intensities and an admittedly large scatter in the measured values, in particular for the weaker peaks of the rectangular phase. Some compensation for this is obtained from the requirement that all three peaks of the rectangular phase, Figure $4 \mathrm{~b}-\mathrm{d}$, should be fitted well by a single molecular length, tilt angle, and azimuthal tilt direction. The fit shown yields a molecular tilt angle of $\theta=(28 \pm 3)^{\circ}$ from the surface normal, in the azimuthal direction of next-nearest neighbors (NNN).

The BR of the hexagonal diffraction peak is shown in Figure 4e. The BR peaks slightly higher than $q_{z}=0 \AA^{-1}$, indicating a small molecular tilt. The fitted tilt angle is $\theta=(7 \pm 3)^{\circ}$, again in the NNN azimuthal direction.

With a molecular tilt of $\theta=28^{\circ}$, the rectangular phase's unit cell in the plane perpendicular to the molecular long axis has $\mathbf{a}_{\perp}=(8.09 \pm 0.01) \AA$ and $\mathbf{b}_{\perp}=(8.46 \pm 0.01) \times \cos 28^{\circ}=$ (7.47 \pm 0.009$) \AA$, close to the width of the anthrone molecule. The molecular area is $A_{\perp}=\mathbf{a}_{\perp} \times \mathbf{b}_{\perp} / 2=(30.2 \pm 0.1)$ $\AA^{2} /$ molecule. The tilt angle of the hexagonal unit cell, $\theta=7^{\circ}$, yields a unit cell in the plane perpendicular to the molecular long axis of dimensions $\mathbf{a}_{\perp}=(5.84 \pm 0.01) \AA$ and $\mathbf{b}_{\perp}=5.84$ $\sqrt{3} \times \cos 7^{\circ}=(10.04 \pm 0.02) \AA$, and a molecular area of $A_{\perp}$ $=(29.3 \pm 0.1) \AA^{2} /$ molecule. Thus, the molecular area of the rectangular phase is larger than that of the hexagonal phase not only in the plane of the substrate but also in the plane perpendicular to the molecular long axis, in spite of the larger tilt angle.

E. Anthralin: Isotherm. The $\pi-A$ isotherm of anthralin on mercury, measured at room temperature, $T=24{ }^{\circ} \mathrm{C}$, is shown in Figure 5. As for all previously discussed molecules, for $A \geq$ $110 \AA^{2} /$ molecule the LF behaves as a dilute $2 \mathrm{D}$ gas of molecules. For $A \lesssim 110 \AA^{2} /$ molecule a steep rise in $\pi$ is observed, from $\pi$ $\approx 5 \mathrm{mN} / \mathrm{m}$ at $A \approx 113 \AA^{2} / \mathrm{molecule}$ to $\pi \approx 40 \mathrm{mN} / \mathrm{m}$ at $A \approx$ $60 \AA^{2} /$ molecule. This fast-increasing region is well fitted by the Vollmer equation (for all points in the range $71 \leq A \leq 450$ $\AA^{2} /$ molecule), as shown in the dashed line in Figure 5, and yields an exclusion area of $A_{0}=(62 \pm 1) \AA^{2} /$ molecule. Similar to the anthrone, $A_{0}$ is smaller than the area of a flat-lying anthracene molecule, $A \gtrsim 90 \AA^{2} /$ molecule, indicating a side-lying orientation of the anthralin molecule in this phase. However, the X-ray data below indicates that the long axis of the side-lying molecule is tilted up from the surface plane so that the length of the molecule, projected onto the mercury surface is $\sim 14 \AA$. This, and the extra $\sim 0.7 \AA$ thickness of the anthralin over that of anthracene due to the oxygen moieties, as obtained from the GID measurements discussed below, yield a calculated molecular area $A_{\text {calcd }}^{\text {ld }}=l \times d \approx 14 \times(3.8+0.7)=63 \AA^{2} /$ molecule

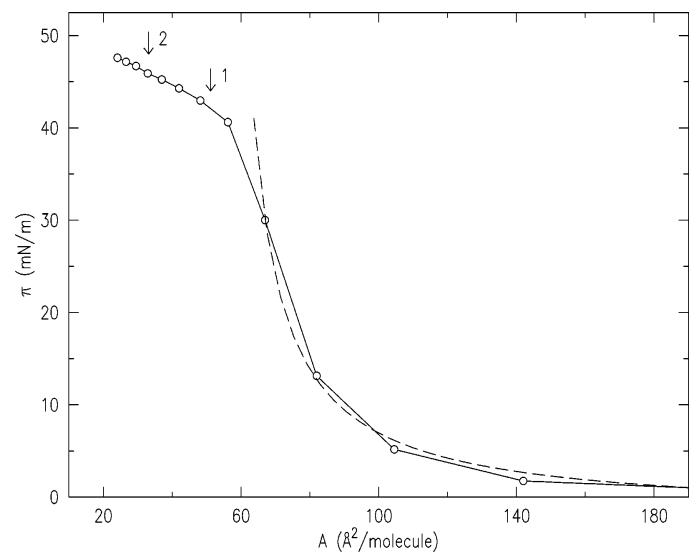

Figure 5. $\pi-A$ isotherm of a LF of anthralin on mercury (circles + solid line). The dashed line is a fit by the Volmer equation, yielding an exclusion area of $A_{0}=62 \AA^{2} /$ molecule. The arrows mark the coverages where $\mathrm{X}$-ray measurements were carried out.
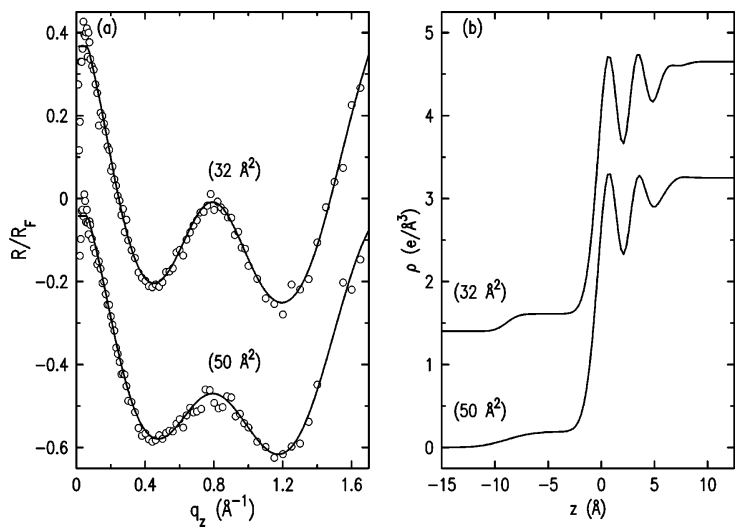

Figure 6. (a) The measured, Fresnel-normalized, X-ray reflectivity curves of anthralin (open circles) on mercury, with their box model fits (lines). Curves are shifted vertically by 0.4 each for clarity. (b) The model density profiles obtained from the fits in part a. The $\mathrm{Hg}$ surface is at $z=0$ and the positive $z$ axis points into the liquid. Curves are shifted by 1.4 for clarity.

for a side-lying anthralin molecule, in excellent agreement with the exclusion area $A_{0}=62 \AA^{2} /$ molecule obtained here.

For $A \lesssim 62 \AA^{2} /$ molecule a plateau is observed in the isotherm. However, the plateau is significantly more sloped than the corresponding almost-horizontal plateau observed in the isotherm of anthrone in Figure 2. The X-ray measurements detailed below indicate that unlike anthrone, where the plateau corresponded to a coexistence region between two different phases, only a single crystalline phase of roughly side-lying (SL) molecules is observed here at both ends of the plateau. This point is discussed in more detail in the next section.

F. Anthralin: Surface-Normal Structure. Figure 6a presents room-temperature $\left(T=24^{\circ} \mathrm{C}\right)$ measured (open circles) and model-fitted (lines) XR curves for the anthralin, at two different nominal coverages. The surface-normal density profiles derived from the fits are shown in Figure 6b. The XR curves shown were measured at a low coverage of $50 \AA^{2} /$ molecule, somewhat below the onset of the plateau, and at a high coverage of $32 \AA^{2} /$ molecule, slightly larger than film collapse. As can be observed, the XR curves are similar, with the same-period fringes, and the only difference is the higher maximumminimum contrast, which is larger at high coverage. This indicates that there is only one phase that gives rise to an observable reflectivity from $A_{0}$ to film collapse. This is in contrast with our conclusions ${ }^{1}$ for anthracene and anthraquinone, 

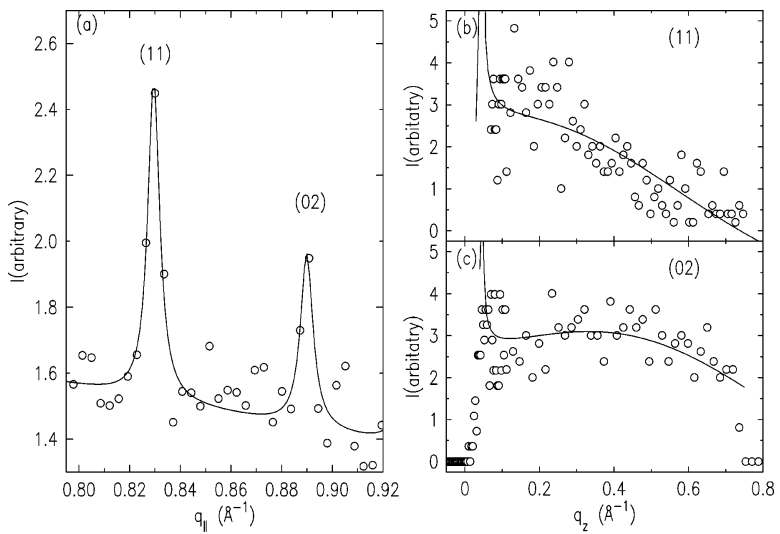

Figure 7. Measured GID (a), BR (b and c, open circles), and model fits (lines) for a LF of anthralin at a coverage of $32 \AA^{\circ} /$ molecule at room temperature. The BR fits yield a tilt angle of $\theta=27^{\circ}$ in the next-nearest neighbor $(\mathrm{NNN})$ direction.

where the low- and high-coverage reflectivity curves were similar but not identical, yielding two different observable phases at low and high coverage. One possible explanation for the observation of a single reflectivity at different converges is if some fraction of the molecules are assumed to aggregate at low coverage into rough structures such as islands. The surface would then be somewhat heterogeneous and would contain a uniform-thickness layer across the full surface area, with a small fraction of rough islands. Similar island phases were found in LFs on water. ${ }^{16}$ The larger apparent roughness observed in the density profile at the monolayer-air interface at $50 \AA^{2} /$ molecule is also consistent with this suggestion. Finally, we note that neither time nor temperature dependencies were observed for the anthralin LF over a period extending from 0.5 to $10 \mathrm{~h}$ after film spreading, and for $T=7,10,20$, and $24{ }^{\circ} \mathrm{C}$.

The fit of the XR curves yields a layer thickness of $(8.9 \pm$ $0.1) \AA$ and electron densities of $(0.19 \pm 0.01)$ and $(0.21 \pm 0.01)$ $\mathrm{e} / \AA^{3}$ at the low and high coverage, respectively. With the anthracene molecule's dimensions, ${ }^{17} 12 \times 7.5 \times 3.8 \AA^{3}$, the fitted layer thickness indicates that the monolayer comprises side-lying molecules. However, as we show in the next section, the GID results reveal that the molecular long axis is tilted up from the surface-parallel orientation, which accounts for the fact that the layer thickness found here is $\sim 1.4 \AA$ larger than the molecular width. The electron density calculated from the layer thickness, exclusion area $A_{0}$, and the 118 electrons of the molecule, $\rho_{\text {calcd }}=118 /(62 \times 8.9)=0.21 \mathrm{e} / \AA^{3}$ is close to the fitted value. This further supports the conclusion that the film is a monolayer of side-lying molecules, rather than a bilayer of flat-lying molecules, which, although consistent with the fitted layer thickness, would have yielded a higher $\rho_{\text {calcd }}=2 \times 118 /$ $(12 \times 7.5 \times 8.9)=0.29 \mathrm{e} / \AA^{3}$

G. Anthralin: Surface-Parallel Structure. The GID measurements on the Anthralin LF were carried out over the range $0.36 \leq q_{\|} \leq 1.71 \AA^{-1}$, at the two coverages studied by XR, 50 and $32 \AA^{2} /$ molecule, as well as at 25.5 and $26.5 \AA^{2} /$ molecule. Although most scans were done at room temperature, $T=24$ ${ }^{\circ} \mathrm{C}$, some were carried out at a lower temperature of $7{ }^{\circ} \mathrm{C}$.

The GID pattern measured at $32 \AA^{2} /$ molecule $2 \mathrm{~h}$ after film spreading (open circles) is shown in Figure 7a, along with its fit (line). The fitting function includes a linear background and a single Lorentzian for each peak. Figure 7a shows two peaks, one at $q_{\|}=(0.830 \pm 0.004) \AA^{-1}$, and the other at $q_{\|}=(0.889$ $\pm 0.004) \AA^{-1}$, which can be indexed as (11) and (02) in a rectangular cell with dimensions $\mathbf{a}=(8.97 \pm 0.04) \AA$ and $\mathbf{b}=$ $(14.15 \pm 0.07) \AA$, with two molecules per unit cell. ${ }^{10}$ The (a)

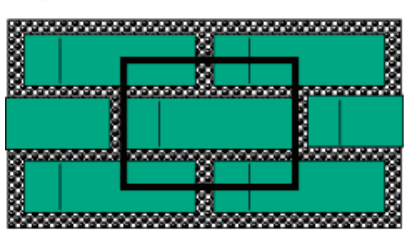

(b)
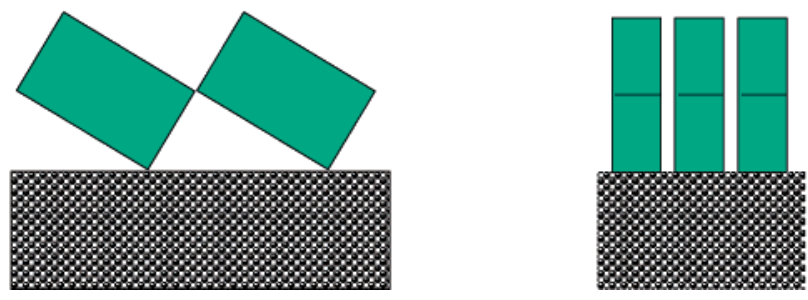

Figure 8. Schematic plot of the packing of anthralin in the LF. The molecule is represented by a rectangle (d). The top (a) and two side (b and c) views of the packing are shown.

resultant area per molecule, $(63.5 \pm 0.4) \AA^{2} /$ molecule, agrees well with the exclusion area, $A_{0} \approx 62 \AA^{2} /$ molecule, obtained from the isotherm. In contrast with the anthrone's SU phase, the present GID pattern does not show any odd $(h+k)$ peaks, indicating a centered unit cell. Within our measurement accuracy, the GID patterns are identical for the four coverages studied, and independent of temperature.

The intensities of the two GID peaks were very low, resulting in a correspondingly low intensity for the Bragg rods, as can be observed in Figure $7 \mathrm{~b}$ and c. Thus, the peak positions and exact shapes of the BRs could not be determined very accurately. Within these constraints, the maxima of the BRs at the two GID peaks are at $q_{z 1}(11) \approx 0.23 \AA^{-1}$ (Figure $7 \mathrm{~b}$ ) and $q_{z 2}(02) \approx 0.45$ $\AA^{-1}$ (Figure $7 \mathrm{c}$ ), that is, $q_{z 2}(02) \approx 2 \times q_{z 1}(11)$. Moreover, in spite of the low intensities, several scans show consistently that the integrated intensity ratio of the peaks $I\left(q_{z 1}\right)(11) \approx 2 \times I\left(q_{z 2^{-}}\right.$ (02)). This intensity ratio, along with the offsets of the BR peaks from $q_{z}=0 \AA^{-1}$, identify a molecular tilt in the NNN direction, ${ }^{10}$ as found above for the SU phase of anthrone. The angle of the molecular tilt from the surface normal is then calculated from the BR peak positions as $\theta=\tan ^{-1}\left[q_{z 2} / q_{\|}(z 2)\right] \approx 26^{\circ}$.

We now focus on the arrangement of the molecules in the unit cell. The long lattice vector, $\mathbf{b}=14.15 \AA$, is longer than the molecular length of the anthralin molecule, $12 \AA$. However, it is very close to the diagonal of the rectangle bounding the three rings: $\sqrt{7.5^{2}+12^{2}}=14.15 \AA$. This coincidence may indicate an uptilt of one end of the molecule from the surface, presumably due to the oxygens, so that the molecule's diagonal, rather than its length, determines the unit cell's length. Given the $7.5 \times 12 \AA^{2}$ dimensions of the molecule, an uptilt of $\sim 23^{\circ}$ will suffice to yield $\mathbf{a}|\mathbf{b}| \approx 14 \AA$. This in turn will yield an average molecular height of $\sim 9.2 \AA$, close to the $8.9 \AA$ monolayer thickness obtained from the XR fits. For side-lying, surface-normal molecules the long axis of which is parallel to b, this would also provide the magnitude and direction of the molecular tilt deduced from the BR measurements in the previous paragraph.

A suggested schematic model for the packing of the anthralin molecules in the LF is shown in Figure 8, depicting the uptilt of the long molecular axis and a possible tilt of the molecular plane from the surface normal. Although this arrangement is in agreement with all measured quantities, and closely resembles the bulk packing of crystalline anthralin ${ }^{18}$ as we discuss below, other packing arrangements may also be possible. 
A determination of the exact ordering (positions and orientations) of the anthralin molecules within the unit cell requires a refinement of a diffraction pattern with a reasonable number of diffraction lines. Because beam damage prevented achieving good statistics even for the two peaks observed, and because higher-order peaks were not observed, a standard structure refinement could not be carried out. However, several plausible suggestions can be made based on the GID and BR patterns observed, and other known properties of the molecule. The oxygen atoms are known to have a high electronegativity (3.46), which, for anthralin, result in a high calculated dipole moment of $\sim 3.6 \mathrm{D}^{19}$ in the direction of the short molecular axis. The strong dipole-dipole interaction may therefore cause an antiparallel orientation of the oxygens in adjacent molecules, with those of one molecule pointing toward the mercury subphase and those of the other pointing away from it. A similar antiparallel orientation of adjacent molecules is found in the bulk packing of anthralin. ${ }^{18} \mathrm{We}$ also note that for anthralin molecules oriented with their long axis roughly in the $\mathbf{b}$ direction, the length of $\mathbf{a}$ is $\sim 1.4 \AA$ larger than the combined thickness of two anthracene molecules, $2 \times 3.8 \AA$. This may be due to the presence of the oxygen moieties of the molecule, the positions of which deviate slightly from the plane of the anthracene backbone. A contribution from a small tilt $\left(<10^{\circ}\right)$ of the molecular plane from the surface normal, similar to that observed in the bulk crystal structure, ${ }^{18}$ may be also included in this increased effective molecular thickness. A small rotation of the molecular plane away from the $\mathbf{b}$ direction, around a surface-normal axis, could also account for the excess length of a. This is particularly appealing because if this rotation is in opposite azimuthal directions for the molecules at the vertices and at the center of the unit cell, a herringbone-like structure, with glide plane symmetry with respect to $\mathbf{b}$ would ensue.

The GID peaks' full width at half-maximum is close to the resolution limit, $\delta q_{\|}=0.006 \AA^{-1}$, and yields a crystalline coherence length of $\xi \geq 1000 \AA$, consistent with the large coherence lengths of other herringbone-packed 2D crystals, ${ }^{10}$ and the rectangular phase of anthrone monolayers, discussed above.

The GID measurements were repeated at $T=24,20$, and 7 ${ }^{\circ} \mathrm{C}$. Thermal expansion, observable as shifts in the peaks' positions, was expected to be similar to that of the herringbone phases of mercury-supported LFs of fatty acids, ${ }^{4}$ alcohols ${ }^{6}$ and biphenyls. ${ }^{20}$ In these cases, an asymmetric thermal expansion was found with no measurable expansion, within our temperature range, in the a direction, and an expansion coefficient of $(\mathrm{d} b / \mathrm{d} T) / b=(6 \pm 3) \times 10^{-4} \mathrm{~K}^{-1}$ in the $\mathbf{b}$ direction. For the Anthralin, no changes were observed, within our experimental accuracy, in the peak positions over the temperature range studied, indicating an expansion coefficient less than $(2.5 \pm$ $0.5) \times 10^{-5} \mathrm{~K}^{-1}$. This high thermal rigidity may be due partly to the strong interactions between the aligned rings of adjacent rigid molecules, and partly to the strong intermolecular $\mathrm{H}$ bonding, ${ }^{18,21,22}$ due to the $\mathrm{O}$ and $\mathrm{OH}$ moieties.

H. Anthralin: Bragg Rods and Molecular Tilt. Figure 7b and $\mathrm{c}$ shows the BR of the two peaks of the anthralin. The fullshape fit of the measured Bragg rods, shown in lines, yield a tilt angle of $\theta=27^{\circ}$ toward the NNN azimuthal direction, in agreement with the conclusions derived in the previous section from the peak positions of the BR along the $q_{z}$ direction. This tilt yields a unit cell in the plane perpendicular to the molecular long axis, with the lattice parameters $\mathbf{a}_{\perp}=(8.97 \pm 0.04) \AA$ and $\mathbf{b}_{\perp}=(14.15 \pm 0.07) \times \cos 27^{\circ}=(12.61 \pm 0.06) \AA$, close to the length of the molecule, as expected. The corresponding area is $A_{\perp}=(56.5 \pm 0.4) \AA^{2} /$ molecule, in close agreement with $A_{\perp \text { calcd }}=12.6 \times(3.8+0.7)=56.7 \AA^{2} /$ molecule, calculated from the molecular dimensions and the extra $\sim 1.4 \AA$ spacing between molecules in the unit cell, derived above from the GID measurements.

Comparing the structure of the anthralin monolayer on mercury with that of the crystalline bulk, ${ }^{18}$ we observe a close general similarity. The unit cell of the bulk phase contains eight molecules, the molecular planes of adjacent molecules are parallel, and the molecules are rotated by $180^{\circ}$ relative to each other so that the oxygens of adjacent molecules point in opposite directions. The molecular long axis is tilted up from the $\mathbf{a}-\mathbf{b}$ plane of the unit cell, and the molecular plane may be tilted away slightly from the normal to that plane. All of these features are also observed for the anthralin LF on mercury as well, although the angles and distances are different. ${ }^{23}$ Such differences are, however, expected, and indeed observed, ${ }^{10}$ when comparing the structure of the bulk with that of a subphasesupported monolayer.

\section{Discussion and Conclusions}

Taken together, the isotherms and X-ray results presented here and in ref 1 for monolayers of anthracene and its derivatives on mercury demonstrate the important influence of the molecule's functional groups, and their positions within the molecule, in the determination of the film structure and the phases observed as a function of coverage.

Both anthracene and anthraquinone exhibit the same structure of flat-lying molecules for molecular areas down to $A \approx 90$ $\AA^{2} /$ molecule, the molecular-plane area of the molecule. Below this $A$ a phase of side-lying molecules is formed, with the molecular plane roughly normal to the surface. Thus, one can conclude that the balance between the two major interactions found to dominate the structure of penatcene on solid surfaces, as discussed in the Introduction of the previous paper, ${ }^{1}$ also dominate the structure of the symmetric molecules. At low coverages the $\pi$ orbital-subphase contact dominates causing the molecules to lie flat on the surface. At high coverages, the $\pi-\pi$ orbital overlap dominates and the molecules are driven into a side-lying orientation. The surface pressure drives the transition from one orientation to the other. The absence of GID peaks for both molecules and in both phases indicates that no long-range in-plane order is established in these films. This again is in line with the conclusion that the epitaxy to the surface's structure dominates the film's structure. ${ }^{1}$ In pentacene on crystalline substrates the film exhibits long-range order commensurate at least in one direction with that of the substrate. ${ }^{24,25}$ For a short-range-ordered liquid metal substrate employed here, the monolayer of the symmetric molecules also exhibits shortrange order only at all coverages.

The oxygen moieties of the anthraquinone do not produce any changes in the structure except for a slightly larger exclusion area. The symmetric positions of the oxygens on carbon nos. 9 and 10 result in the absence of a net dipole moment, which does exist for both the anthrone and anthralin, where the oxygens are attached to one side of the molecule only. Indeed, the LFs of the asymmetric anthrone and anthralin molecules show structures and phase sequences that differ greatly from those of the symmetric molecules. The exclusion areas deduced from the isotherms indicate that at the steep rise in the isotherm both molecules are roughly side-lying. For the anthrone, this phase does not exhibit long-range in-plane order. At high coverage, the anthrone exhibits two coexisting phases of standing-up molecules, one having a noncentered rectangular packing with 
molecules tilted by $27^{\circ}$ in the NNN direction, and a large, $>1000 \AA$ crystalline coherence length, and the other an hexagonal phase with a much smaller tilt, $7^{\circ}$, in the same direction, and a smaller coherence length of $\sim 200 \AA$ only. The anthralin exhibits only a single phase both at low and high coverage: the molecules are side-lying, with the molecular axis tilted up from the surface. The average tilt angle, obtained from several different measurements, is $(27 \pm 2)^{\circ}$. Unlike the sidelying phase of the anthrone, for the anthralin long-range inplane order is found, with a coherence length of $>1000 \AA$.

The different behavior of the last two molecules from that of the first two is very likely related to the positions of the oxygens. Their asymmetric positions in the last two molecules induce a strong dipole moment, calculated to be 3.3 and 3.6 $\mathrm{D}^{19}$ for the anthrone and anthralin, respectively, along the short axis of the molecules. This adds a strong dipole-dipole interaction, creating, apparently, a new balance between the molecule-molecule and molecule-subphase interactions, and, consequently, different structures emerge. The emergence of in-plane long-range order in these films, where none exists in either the subphase or in the symmetric anthracene and anthraquinone, can also be assigned to the strong dipole-dipole intermolecular interaction in these asymmetric molecules. The difference between the anthrone, which exhibits standing-up phases, and the anthralin, which does not, is more subtle, and can be speculated to be related to the ability of the anthralin's three oxygen moieties to form hydrogen bonds among themselves. The bulk crystal structure of these two molecules is also different, with some of the differences paralleling those found here for the LFs. Studies similar to those presented here on anthracene derivatives, having other chemical side groups, varying in number and position on the molecule, should provide deeper insight into the details of the interactions controlling the structure of these LFs.

Acknowledgment. Support to M.D. by the U.S.-Israel Binational Science Foundation, Jerusalem and to P.S.P. by the NSF (grant No. DMR-0124936) is gratefully acknowledged. We thank Hanna Weitman for useful discussions, and Yaakov Kimhi for the dipole moment calculations. BNL is supported by U.S. DOE Contract No. DE-AC02-98CH10886.

\section{References and Notes}

(1) Tamam, L.; Kraack, H.; Sloutskin, E.; Ocko, B. M.; Pershan, P. S.; Ofer, E.; Deutsch, M. J. Phys. Chem. C 2007, 111, 2573.

(2) Streitwieser, A; Heathcock, C. H.; Kosower, E. M. Introduction to Organic Chemistry, 4th ed.; Prentice Hall: New Jersey, 1998.

(3) (a) Kraack, H.; Ocko, B. M.; Pershan, P. S.; Deutsch, M. Science 2002, 298, 1404. (b) Kraack, H.; Deutsch, M.; Ocko, B. M.; Pershan, P. S. Nucl. Instrum. Methods Phys. Res., Sect. B 2003, 200, 363.

(4) Kraack, H.; Ocko, B. M.; Pershan, P. S.; Sloutskin, E.; Tamam, L.; Deutsch M. Langmuir 2004, 20, 5375.

(5) Kraack, H.; Ocko, B. M.; Pershan, P. S.; Sloutskin, E.; Deutsch M. J. Chem. Phys. 2003, 119, 10339.

(6) Kraack, H.; Ocko, B. M.; Pershan, P. S.; Sloutskin, E.; Tamam, L.; Deutsch M. Langmuir 2004, 20, 5386.

(7) Ocko, B. M.; Kraack, H.; Pershan, P. S.; Sloutskin, E.; Tamam, L.; Deutsch M. Phys. Rev. Lett. 2004, 94, 017802.

(8) Jensen, T. R.; Kjaer, K. In Novel Methods to Study Interfacial Layers; Möbius, D., Miller, R. Eds.; Elsevier: Amsterdam, 2001.

(9) (a) Flack, H. D. Philos. Trans. R. Soc. London, Ser. A 1970, 266,

561. (b) Reynolds, P. A. Acta. Crystallogr., Sect. A 1975, 31, 80.

(10) Kaganer, V. M.; Möhwald, H.; Dutta, P. Rev. Mod. Phys. 1999 , 71,779 .

(11) Als-Nielsen, J.; Jacquemain, D.; Kjaer, K.; Leveiller, F.; Lahav, M.; Leiserowitz, L. Phys. Rep. 1994, 246, 252.

(12) Rapaport, H.; Kuzmenko, I.; Lafonte, S.; Kjaer, K.; Howes, P. W.; Als-Nielsen, J.; Lahav M.; Leiserowitz, L. Biophys. J. 2001, 81, 2729.

(13) Sönchen, S.; Hänel, K.; Birkner, A.; Witte, G.; Wöll, C. Chem Mater. 2005, 17, 5297.

(14) Srivastava, S. N. Acta Crystallogr. 1964, 17, 851

(15) Ocko, B. M.; Wu, X. Z.; Sirota, E. B.; Sinha, S. K.; Gang, O.; Deutsch, M. Phys. Rev. E. 1996, 55, 3164

(16) Grayer Wolf, S.; Leiserowitz, L.; Lahav, M.; Deutsch, M.; Kjaer, K.; Als-Nielsen, J. Nature 1987, 328, 63.

(17) Sander, L. C.; Wise, A. Polycyclic Aromatic Hydrocarbon Structure Index; NIST Special Publication 922; NIST: Gaithersburg, MD, 1997.

(18) Ahmed, F. R Acta Crystallogr., Sect. B. 1980, 38, 3184.

(19) The dipole moments were calculated using the Materials Studio program.

(20) Tamam, L.; Kraack, H.; Sloutskin, E.; Ulman, A.; Pershan, P. S.; Deutsch, M.; Ocko, B. M. J. Phys. Chem. B 2005, 109, 12534.

(21) Andersen, K. B.; Langgard, M.; Larsen, J. S. J. Mol. Struct. 1999, $475,131$.

(22) (a) Andersen, K. B.; Spanget-Larsen, J. Acta Crystallogr., Sect. A 1997, 53, 2615. (b) Moller, S.; Andersen, K. B.; Spanget-Larsen, J.; Waluk, J. Chem. Phys. Lett. 1998, 291, 51.

(23) Note that the $\mathbf{a}$ and $\mathbf{b}$ axes are interchanged in the LF as compared to those of the bulk crystal structure, as given in ref 18 .

(24) Schreiber, F. Phys. Status Solidi A 2004, 201, 1037.

(25) Witte, G.; Wöll, Ch. J. Mater. Res. 2004, 19, 1889. 